\title{
Non-Hermitian quantum mechanics in non-commutative space
}

\author{
Pulak Ranjan Gir:* \\ Theory Division, Saha Institute of Nuclear Physics, 1/AF Bidhannagar, Calcutta 700064, India \\ P Roy \\ Physics and applied mathematics Unit, Indian Statistical Institute, kolkata 700108, India
}

(Dated: November 3, 2018)

\begin{abstract}
We study non Hermitian quantum systems in noncommutative space as well as a $\mathcal{P} \mathcal{T}$-symmetric deformation of this space. Specifically, a $\mathcal{P} \mathcal{T}$-symmetric harmonic oscillator together with $i C\left(x_{1}+\right.$ $x_{2}$ ) interaction is discussed in this space and solutions are obtained. It is shown that in the $\mathcal{P} \mathcal{T}$ deformed noncommutative space the Hamiltonian may or may not possess real eigenvalues depending on the choice of the noncommutative parameters. However, it is shown that in standard noncommutative space, the $i C\left(x_{1}+x_{2}\right)$ interaction generates only real eigenvalues despite the fact that the Hamiltonian is not $\mathcal{P} \mathcal{T}$-symmetric. A complex interacting anisotropic oscillator system has also been discussed.

PACS numbers: 03.65.-w, 03.65.Db, 03.65.Ta
\end{abstract}

\section{INTRODUCTION}

The fact, that the spacetime could be noncommutative [1, 2] at very small length scale, received much attention in recent years. The typical length scale relevant for noncommutative physics is Planck scale. However, one usually looks for phenomenological consequence of noncommutativity in quantum mechanical regime. On the other hand, quantum mechanics is a simple platform for studying noncommutative physics and testing [3] the effect of noncommtativity. In noncommutative spacetime the ordinary product is replaced by a star product of the form

$$
\psi(x) \star \phi(x)=\left.\psi(x) \exp \left\{-i \theta^{\mu \nu} \frac{\overleftarrow{\partial}}{\partial x^{\mu}} \frac{\vec{\partial}}{\partial y^{\nu}}\right\} \phi(y)\right|_{x=y},
$$

where the parameter $\theta^{\mu \nu}, \mu, \nu=1,2,3$, is antisymmetric with respect to $\mu$ and $\nu$. It is easy to check that, (1) leads to the coordinate noncommutativity $\left[x^{\mu}, x^{\nu}\right]=i \theta^{\mu \nu}$, by simply replacing $\psi=x^{\mu}, \phi=x^{\nu}$ in (1). This type of noncommutativity has been used in various fields like quantum field theory, string theory, quantum mechanics $[4,5,6]$.

On the other hand in recent years there have been a great deal of interest in the sudy of non Hermtian (as well as $\mathcal{P} \mathcal{T}$ symmetric) quantum mechanics [10, 11, 12, 13, 14]. Recently there has also been attempts to create $\mathcal{P} \mathcal{T}$ symmetric systems in the laboratory [15]. Here our objective is to study non Hermitian quantum mechanics in non commutative space. To be more specific we shall study models which are $\mathcal{P} \mathcal{T}$ symmetric as well as those which are non Hermitian and non $\mathcal{P} \mathcal{T}$ symmetric. It may be noted that noncommutative physics, defined by

\footnotetext{
*Electronic address: pulakranjan.giri@saha.ac.in
}

${ }^{\dagger}$ Electronic address: pinaki@isical.ac.in $\left[x^{\mu}, x^{\nu}\right]=i \theta^{\mu \nu}$, is not $\mathcal{P} \mathcal{T}$-symmetric. We see that $\theta^{\mu \nu}$ does not change under $\mathcal{P} \mathcal{T}$ transformation. The fact that $\theta^{\mu \nu}$ does not change under $\mathcal{P}$ and $\mathcal{T}$, hence under $\mathcal{P} \mathcal{T}$ has been pointed out in [16]. However, in [16] certain transformation properties have been imposed so that the theory achieve a certain symmetry.

In this article we shall first consider a $\mathcal{P} \mathcal{T}$ symmetric deformation of non commutative space and examine some models in this space. Secondly we shall also examine non Hermitian (but not necessarily $\mathcal{P} \mathcal{T}$ symmetric) models defined in standard non commutative space. It will be seen that in quite a few cases it is possible to obtain real spectrum despite the models being non Hermitian. We consider our problem on a plane. The $x_{3}$ coordinate commutes with the coordinates of the plane and the dynamics along the $x_{3}$ direction is taken to free throughout our discussion.

The remainder of this article is organized as follows: In the next section we propose a $\mathcal{P} \mathcal{T}$-symmetric generalization of the noncommutative quantum mechanics. As a simple example we then discuss the isotropic oscillator in this $\mathcal{P} \mathcal{T}$-symmetric noncommutative space. In section [III we discuss a $\mathcal{P} \mathcal{T}$-symmetric displaced oscillator in our formulation of noncommutative space and solve it. Here we also discuss the same model in the standard non commutative space. A non-hermitian oscillator is then discussed in IV] Finally, section $\mathrm{V}$ is devoted to a conclusion.

\section{II. $\mathcal{P} \mathcal{T}$-SYMMETRIC DEFORMATION}

In this section we discuss the noncommutative quantum mechanics (QM), where the noncommutativity respects $\mathcal{P} \mathcal{T}$ symmetry, unlike the the standard formalism, where the noncommutative algebra [7]

$$
\begin{array}{r}
{\left[\hat{x_{\mu}}, \hat{x_{\nu}}\right]=i 2 \theta_{\mu \nu}, \quad\left[\hat{p_{\mu}}, \hat{p_{\nu}}\right]=i 2 \hat{\theta_{\mu \nu}},} \\
{\left[\hat{x_{\mu}}, \hat{p_{\nu}}\right]=i \hbar\left(\hat{\delta_{\mu \nu}}+\theta_{\mu}^{\beta} \hat{\theta_{\nu \beta}}\right),}
\end{array}
$$


does not respect $\mathcal{P} \mathcal{T}$-symmetry. Note that $\mathcal{P} \mathcal{T}$ symmetry is broken by the first two relations of the algebra (2) but the third one respects the symmetry

$$
\begin{aligned}
& \mathcal{P} \mathcal{T}\left[\hat{x_{\mu}}, \hat{x_{\nu}}\right] \mathcal{P} \mathcal{T}^{-1} \neq \mathcal{P} \mathcal{T} i 2 \theta_{\mu \nu} \mathcal{P} \mathcal{T}^{-1}, \\
& \mathcal{P} \mathcal{T}\left[\hat{p_{\mu}}, \hat{p_{\nu}}\right] \mathcal{P} \mathcal{T}^{-1} \neq \mathcal{P} \mathcal{T} i 2 \theta_{\mu \nu} \mathcal{P} \mathcal{T}^{-1}, \\
& \mathcal{P} \mathcal{T}\left[\hat{x_{\mu}}, \hat{p_{\nu}}\right] \mathcal{P} \mathcal{T}^{-1}=\mathcal{P} \mathcal{T} i \hbar\left(\delta_{\mu \nu}+\theta_{\mu}^{\beta} \theta_{\nu \beta}\right) \mathcal{P} \mathcal{T}^{-1}
\end{aligned}
$$

It is also clear from the representation

$$
\hat{x_{\mu}}=x_{\mu}-\theta_{\mu \nu} p^{\nu}, \hat{p_{\mu}}=p_{\mu}+\hat{\theta_{\mu \nu}} x^{\nu},
$$

of the noncommutative coordinates on the phase space that they do not behave as the commutative coordinates

$$
\mathcal{P} \mathcal{T} \hat{x_{\mu}} \mathcal{P} \mathcal{T}^{-1} \neq-\hat{x_{\mu}}, \quad \mathcal{P} \mathcal{T} \hat{p_{\mu}} \mathcal{P} \mathcal{T}^{-1} \neq \hat{p_{\mu}}
$$

under $\mathcal{P} \mathcal{T}$ transformation. We can however consider a noncommutative formulation where the commutator algebra are $\mathcal{P} \mathcal{T}$-symmetric. This can be done by replacing $\theta_{\mu \nu} \rightarrow i \theta_{\mu \nu}, \hat{\theta_{\mu \nu}} \rightarrow i \hat{\theta_{\mu \nu}}$ in the algebra (2)

$$
\begin{array}{r}
{\left[\hat{x_{\mu}}, \hat{x_{\nu}}\right]=-2 \theta_{\mu \nu},\left[\hat{p_{\mu}}, \hat{p_{\nu}}\right]=-2 \hat{\theta_{\mu \nu}},} \\
{\left[\hat{x_{\mu}}, \hat{p_{\nu}}\right]=i \hbar\left(\hat{\delta_{\mu \nu}}-\theta_{\mu}^{\beta} \hat{\theta_{\nu \beta}}\right),}
\end{array}
$$

It is useful to consider the same representation (4) but with the replacement $\theta_{\mu \nu} \rightarrow i \theta_{\mu \nu}, \hat{\theta_{\mu \nu}} \rightarrow i \hat{\theta_{\mu \nu}}$ as

$$
\hat{x_{\mu}}=x_{\mu}-i \theta_{\mu \nu} p^{\nu}, \hat{p_{\mu}}=p_{\mu}+i \hat{\theta_{\mu \nu}} x^{\nu},
$$

which transforms in the same way as the ordinary coordinate and momentum under $\mathcal{P} \mathcal{T}$-transformation. It is now obvious that any quantum mechanical system which is $\mathcal{P} \mathcal{T}$-symmetric in commutative space will remain $\mathcal{P} \mathcal{T}$ symmetric when considered in the noncommutative space given by the algebra (6). To discuss the QM in this formulation we consider the isotropic oscillator on a plane.

$$
H=\frac{1}{2 m}\left(p_{1}^{2}+p_{2}^{2}\right)+\frac{1}{2} m \omega^{2}\left(x_{1}^{2}+x_{2}^{2}\right),
$$

Eq. (8) is both Hermitian and $\mathcal{P} \mathcal{T}$-symmetric. The corresponding noncommutative counterpart

$$
\hat{H}=\frac{1}{2 m}\left({\hat{p_{1}}}^{2}+{\hat{p_{2}}}^{2}\right)+\frac{1}{2} m \omega^{2}\left({\hat{x_{1}}}^{2}+{\hat{x_{2}}}^{2}\right),
$$

then takes the form

$$
\begin{aligned}
H_{\mathcal{P} \mathcal{T}} & =\frac{1}{2 M_{\mathcal{P} \mathcal{T}}}\left(p_{1}^{2}+p_{2}^{2}\right)+\frac{1}{2} M_{\mathcal{P} \mathcal{T}} \Omega_{\mathcal{P} \mathcal{T}}^{2}\left(x_{1}^{1}+x_{2}^{2}\right) \\
& -i S_{\mathcal{P} \mathcal{T}} L_{z}
\end{aligned}
$$

where $1 / M_{\mathcal{P} \mathcal{T}}=1 / m-m \omega^{2} \theta^{2}, S_{\mathcal{P} \mathcal{T}}=\left(m \omega^{2} \theta+\hat{\theta} / m\right)$, and $\Omega_{\mathcal{P} \mathcal{T}}=\sqrt{\left(1 / m-m \omega^{2} \theta^{2}\right)\left(m \omega^{2}-\hat{\theta}^{2} / m\right)}$. Note that, in order to get (10) we used the relation (77) with $\theta_{12}=$ $\theta, \hat{\theta_{12}}=\hat{\theta}$ and $\mu, \nu=1,2$. It is possible to evaluate the spectrum of the Hamiltonian (10) for a special case $S_{\mathcal{P} \mathcal{T}}=0$, which makes it isotropic. The eigenvalues are

$$
E_{\mathcal{P} \mathcal{T}}=\Omega_{\mathcal{P} \mathcal{T}}\left(n^{+}+n^{-}+1\right)
$$

where $n^{ \pm} \in \mathbb{N}, \Omega_{\mathcal{P} \mathcal{T}}=\omega\left(1-m^{2} \omega^{2} \theta^{2}\right)=\omega(1+\theta \hat{\theta})$. However for general case, $S_{\mathcal{P} \mathcal{T}} \neq 0$, although the Hamiltonian (10) is $\mathcal{P} \mathcal{T}$-symmetric the solution of the corresponding eigenvalue problem can be complex. It is possible to write (10) as a sum of two separate one dimensional harmonic oscillators with frequencies $\Omega_{\mathcal{P} \mathcal{T}}+i S_{\mathcal{P} \mathcal{T}}$ and $\Omega_{\mathcal{P} \mathcal{T}}-i S_{\mathcal{P} \mathcal{T}}$,

$$
\begin{aligned}
H_{\mathcal{P} \mathcal{T}}= & \Omega_{\mathcal{P} \mathcal{T}}\left(a_{\mathcal{P} \mathcal{T}}^{\dagger} a_{\mathcal{P} \mathcal{T}}+b_{\mathcal{P} \mathcal{T}}^{\dagger} b_{\mathcal{P} \mathcal{T}}+1\right) \\
& +i S_{\mathcal{P} \mathcal{T}}\left(a_{\mathcal{P} \mathcal{T}}^{\dagger} a_{\mathcal{P} \mathcal{T}}-b_{\mathcal{P} \mathcal{T}}^{\dagger} b_{\mathcal{P} \mathcal{T}}\right) .
\end{aligned}
$$

Here the annihilation operators $a_{\mathcal{P} \mathcal{T}}$ and $b_{\mathcal{P} \mathcal{T}}$ are

$$
\begin{aligned}
& a_{\mathcal{P} \mathcal{T}}=\frac{\left[\left(p_{1}+i p_{2}\right)-i M_{\mathcal{P} \mathcal{T}} \Omega_{\mathcal{P} \mathcal{T}}\left(x_{1}+i x_{2}\right)\right]}{2 \sqrt{M_{\mathcal{P} \mathcal{T}} \Omega_{\mathcal{P} \mathcal{T}}}}, \\
& b_{\mathcal{P} \mathcal{T}}=\frac{\left[\left(p_{1}-i p_{2}\right)-i M_{\mathcal{P} \mathcal{T}} \Omega_{\mathcal{P} \mathcal{T}}\left(x_{1}-i x_{2}\right)\right]}{2 \sqrt{M_{\mathcal{P} \mathcal{T}} \Omega_{\mathcal{P} \mathcal{T}}}} .
\end{aligned}
$$

The corresponding creation operators $a_{\mathcal{P} \mathcal{T}}^{\dagger}, b_{\mathcal{P} \mathcal{T}}^{\dagger}$ together with the annihilation operators satisfy the usual commutation relations ( $\hbar=1$ unit is used)

$$
\left[a_{\mathcal{P} \mathcal{T}}, a_{\mathcal{P} \mathcal{T}}^{\dagger}\right]=\left[b_{\mathcal{P} \mathcal{T}}, b_{\mathcal{P} \mathcal{T}}^{\dagger}\right]=1
$$

all other commutators are zero. The number operators $\mathcal{N}_{\mathcal{P} \mathcal{T}}^{+}=a_{\mathcal{P} \mathcal{T}}^{\dagger} a_{\mathcal{P} \mathcal{T}}$ and $\mathcal{N}_{\mathcal{P} \mathcal{T}}^{-}=b_{\mathcal{P} \mathcal{T}}^{\dagger} b_{\mathcal{P} \mathcal{T}}$ satisfy the eigenvalue equation $\mathcal{N}_{\mathcal{P} \mathcal{T}}^{ \pm}\left|n^{+}, n^{-}\right\rangle=n^{ \pm}\left|n^{+}, n^{-}\right\rangle$with $n^{ \pm} \in \mathbb{N}_{0}$. The exact eigenvalue for the Hamiltonian (12) can be found as

$$
E_{\mathcal{P} \mathcal{T}}=\Omega_{\mathcal{P} \mathcal{T}}\left(n^{+}+n^{-}+1\right)+i S_{\mathcal{P} \mathcal{T}}\left(n^{+}-n^{-}\right)
$$

Note that the eigenvalue is real only for $n^{+}=n^{-}=n$. In that case $E_{\mathcal{P} \mathcal{T}}=\Omega_{\mathcal{P} \mathcal{T}}(2 n+1)$.

It would be informative at this point to mention the result of isotropic oscillator (8) studied on the standard noncommutative space [8] defined by (2). The 2dimensional isotropic oscillator on a plane with only noncommutative coordinates becomes anisotropic with two different frequencies. Even for both the coordinates and momenta to be noncommutative like (2), the oscillator becomes anisotropic with completely real eigenvalues [9]

$$
E_{N C}=\Omega_{N C}\left(n^{+}+n^{-}+1\right)+S_{N C}\left(n^{+}-n^{-}\right) .
$$

where, $\Omega_{N C}=\sqrt{\left(1 / m+m \omega^{2} \theta^{2}\right)\left(m \omega^{2}+\hat{\theta}^{2} / m\right)}$ and $S_{N C}=\left(m \omega^{2} \theta+\hat{\theta} / m\right)$ and $1 / M_{N C}=1 / m+m \omega^{2} \theta^{2}$. To obtain the above result for $E_{N C}$, the relation (44), with $\theta_{12}=\theta, \hat{\theta_{12}}=\hat{\theta}$ and $\mu, \nu=1,2$ has been used. 


\section{III. $\mathcal{P}$ T-SYMMETRIC OSCILLATOR}

In this section, we consider a $\mathcal{P} \mathcal{T}$-symmetric oscillator given by the Hamiltonian

$$
\begin{aligned}
H_{\mathcal{P} \mathcal{T}} & =\frac{1}{2 m}\left(p_{1}^{2}+p_{2}^{2}\right)+\frac{1}{2} m \omega^{2}\left(x_{1}^{2}+x_{2}^{2}\right) \\
& +i C\left(x_{1}+x_{2}\right)
\end{aligned}
$$

where the real constant $C$ is the strength of $\mathcal{P} \mathcal{T}$ symmetric interaction. It has real eigenvalues [10] with the ground state shifted. The noncommutative version defined as

$$
\begin{aligned}
\hat{H_{\mathcal{P} \mathcal{T}}} & =\frac{1}{2 m}\left({\hat{p_{1}}}^{2}+{\hat{p_{2}}}^{2}\right)+\frac{1}{2} m \omega^{2}\left({\hat{x_{1}}}^{2}+{\hat{x_{2}}}^{2}\right) \\
& +i C\left(\hat{x_{1}}+\hat{x_{2}}\right)
\end{aligned}
$$

is $\mathcal{P} \mathcal{T}$-symmetric, $\mathcal{P} \mathcal{T} \hat{H_{\mathcal{P}} \mathcal{T}} \mathcal{P} \mathcal{T}^{-1}=\hat{H_{\mathcal{P}} \mathcal{T}}$. Note that here we used the algebra (6) and the representation (7). In terms of commutative coordinates (19) can be written as

$$
\begin{aligned}
\hat{H_{\mathcal{P} \mathcal{T}}} & =\frac{1}{2 M_{\mathcal{P} \mathcal{T}}}\left(p_{1}^{2}+p_{2}^{2}\right)+\frac{1}{2} M_{\mathcal{P} \mathcal{T}} \Omega_{\mathcal{P} \mathcal{T}}^{2}\left(x_{1}^{2}+x_{2}^{2}\right) \\
& -i S_{\mathcal{P} \mathcal{T}} L_{z}+i C\left(x_{1}+x_{2}\right)-C \theta\left(p_{1}-p_{2}\right)
\end{aligned}
$$

We first solve the problem (20) for a special case $S_{\mathcal{P} \mathcal{T}}=$ 0 . We use the following transformation

$$
\begin{aligned}
Q_{1} & =x_{1}+\frac{i C}{M_{\mathcal{P} \mathcal{T}} \Omega_{\mathcal{P} \mathcal{T}}^{2}}, \quad Q_{2}=x_{2}+\frac{i C}{M_{\mathcal{P} \mathcal{T}} \Omega_{\mathcal{P} \mathcal{T}}^{2}} \\
P_{1} & =p_{1}-C \theta M_{\mathcal{P} \mathcal{T}}, \quad P_{2}=p_{2}+C \theta M_{\mathcal{P} \mathcal{T}}
\end{aligned}
$$

on (20), which then becomes an isotropic oscillator with a constant shift,

$\hat{H_{\mathcal{P} \mathcal{T}}}=\frac{1}{2 M_{\mathcal{P} \mathcal{T}}}\left(P_{1}^{2}+P_{2}^{2}\right)+\frac{1}{2} M_{\mathcal{P} \mathcal{T}} \Omega_{\mathcal{P} \mathcal{T}}^{2}\left(Q_{1}^{2}+Q_{2}^{2}\right)+E_{0}(22)$

where the constant is $E_{0}=\frac{C^{2}}{M_{\mathcal{P} \mathcal{T}} \Omega_{\mathcal{P} \mathcal{T}}^{2}}-C^{2} \theta^{2} M_{\mathcal{P} \mathcal{T}}$. The eigenvalue is readily obtained to be

$$
\hat{E_{\mathcal{P} \mathcal{T}}}=\Omega_{\mathcal{P} \mathcal{T}}\left(n^{+}+n^{-}+1\right)+E_{0},
$$

However for general case, $S_{\mathcal{P} \mathcal{T}} \neq 0$, although the Hamiltonian (20) is $\mathcal{P} \mathcal{T}$-symmetric, the solution of the corresponding eigenvalue problem can be complex. To solve the eigenvalue problem we make the following transformation

$$
\begin{aligned}
Q_{1} & =x_{1}+i \lambda, Q_{2}=x_{2}+i \lambda, \\
P_{1} & =p_{1}+\beta, P_{2}=p_{2}-\beta,
\end{aligned}
$$

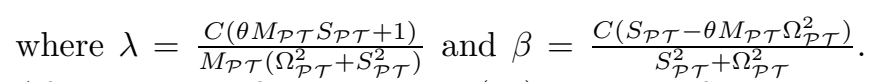
After this transformation Eq. (20) takes the form

$$
\begin{aligned}
\hat{H_{\mathcal{P} \mathcal{T}}} & =\frac{1}{2 M_{\mathcal{P} \mathcal{T}}}\left(P_{1}^{2}+P_{2}^{2}\right)+\frac{1}{2} M_{\mathcal{P} \mathcal{T}} \Omega_{\mathcal{P} \mathcal{T}}^{2}\left(Q_{1}^{2}+Q_{2}^{2}\right) \\
& -i S_{\mathcal{P} \mathcal{T}}\left(Q_{1} P_{2}-Q_{2} P_{1}\right)+\hat{E}_{0},
\end{aligned}
$$

where $\hat{E}_{0}=\beta^{2} / M_{\mathcal{P} T}-M_{\mathcal{P} T} \Omega_{\mathcal{P} T}^{2} \lambda^{2}-2 S_{\mathcal{P} T} \lambda \beta+2 C \lambda+$ $2 C \theta \beta$. The exact eigenvalues for the Hamiltonian (20) can be found as

$$
\hat{E_{\mathcal{P}} \mathcal{T}}=\Omega_{\mathcal{P} \mathcal{T}}\left(n^{+}+n^{-}+1\right)+i S_{\mathcal{P} \mathcal{T}}\left(n^{+}-n^{-}\right)+\hat{E_{0}}
$$

Note that the eigenvalues are real only for $n^{+}=n^{-}=n$, in that case $\hat{E_{\mathcal{P} \mathcal{T}}}=\Omega_{\mathcal{P} \mathcal{T}}(2 n+1)+\hat{E_{0}}$. One can see that the limit $\hat{\theta} \rightarrow 0$ of Eq. (27) can be taken smoothly.

Note that although (27) respects $\mathcal{P} \mathcal{T}$-symmetry, it becomes complex in general. It would now be interesting to see what happens if we consider the standard noncommutativity, given by (2), to study the $\mathcal{P} \mathcal{T}$-symmetric Hamiltonian (18). This can be simply achieved by replacing $i \theta \rightarrow \theta$ and $i \hat{\theta} \rightarrow \hat{\theta}$ from Eq. (20) onwards. It may be seen that in this case the Hamiltonian (18) is non Hermitian and non $\mathcal{P} \mathcal{T}$ symmetric. The eigenvalue are then given by

$$
\hat{E_{N C}}=\Omega_{N C}\left(n^{+}+n^{-}+1\right)+S_{N C}\left(n^{+}-n^{-}\right)+\epsilon_{N C}(28)
$$

where, $\epsilon_{N C}$

$$
\begin{aligned}
& =-\frac{C^{2}\left(S_{N C}-\theta M_{N C} \Omega_{N C}^{2}\right)^{2}}{\left(-S_{N C}^{2}+\Omega_{N C}^{2}\right)^{2} M_{N C}}-\frac{C^{2} \Omega_{N C}^{2}\left(1-\theta M_{N C} S_{N C}\right)^{2}}{M_{N C}\left(\Omega_{N C}^{2}-S_{N C}^{2}\right)^{2}} \\
& +\frac{2 C^{2} S_{N C}\left(1-\theta M_{N C} S_{N C}\right)\left(S_{N C}-\theta M_{N C} \Omega_{N C}\right)}{M_{N C}\left(\Omega_{N C}^{2}-S_{N C}^{2}\right)} \\
& +\frac{2 C^{2}\left(1-\theta M_{N C} S_{N C}\right)}{M_{N C}\left(\Omega_{N C}^{2}-S_{N C}^{2}\right)}-\frac{2 C^{2} \theta\left(S_{N C}-\theta M_{N C} \Omega_{N C}^{2}\right)}{\left(\Omega_{N C}^{2}-S_{N C}^{2}\right)}
\end{aligned}
$$

Note that, this time the eigenvalues (28) are completely real.

\section{ANISOTROPIC OSCILLATOR WITH NON-HERMITIAN COUPLING}

We consider anisotropic oscillator on a plane with a non-hermitian coupling of the form $\sim i x_{1} x_{2}$. The Hamiltonian we consider is of the form [17]

$$
H=\frac{1}{2} \sum_{i=1}^{2}\left(p_{i}^{2}+C_{i} x_{i}^{2}\right)+\frac{1}{2} i C_{3} x_{1} x_{2}
$$

The Hamiltonian (29) is not hermitian $H^{\dagger} \neq H$. We now consider this Hamiltonian in noncommutative space. It is however better to follow the transformation of Ref. [17], which is of the form

$$
\begin{aligned}
X & =\alpha_{1} x_{1}+x_{2}, \quad Y=\alpha_{2} x_{1}+x_{2}, \\
P_{X} & =\frac{p_{1}-\alpha_{2} p_{2}}{\alpha_{1}-\alpha_{2}}, P_{Y}=\frac{p_{1}-\alpha_{1} p_{2}}{\alpha_{2}-\alpha_{1}} .
\end{aligned}
$$

where $\alpha_{1}=\left(\left(C_{1}^{2}-C_{2}^{2}\right)-\sqrt{\left(C_{1}^{2}-C_{2}^{2}\right)^{2}-C_{3}^{2}}\right) /\left(i C_{3}\right)$ and $\alpha_{1}=\left(\left(C_{1}^{2}-C_{2}^{2}\right)+\sqrt{\left(C_{1}^{2}-C_{2}^{2}\right)^{2}-C_{3}^{2}}\right) /\left(i C_{3}\right)$. 
Note that the new variables satisfy the usual commutation rules $\left[X, P_{X}\right]=\left[Y, P_{Y}\right]=i \hbar$. All other commutators being zero. In terms of the new canonical variables $X, Y, P_{X}, P_{Y}$, the Hamiltonian (29) reads as

$$
H=e^{K} H_{K}+e^{-K} H_{-K},
$$

with the two separate one dimensional Hamiltonians

$$
H_{K}=\frac{{\tilde{P_{X}}}^{2}}{2 m}+\frac{1}{2} m \Omega^{2} \tilde{X}^{2}, H_{-K}=\frac{{\tilde{P_{Y}}}^{2}}{2 m}+\frac{1}{2} m \Omega^{2} \tilde{Y}^{2},
$$

where the new canonical phase space coordinates are

$$
\begin{aligned}
\tilde{P_{X}} & =e^{-K / 2} \sqrt[4]{\frac{1+\alpha_{1}^{2}}{1+\alpha_{2}^{2}}} P_{X}, \tilde{P}_{Y}=e \sqrt{K / 2} \sqrt[4]{\frac{1+\alpha_{2}^{2}}{1+\alpha_{1}^{2}}} P_{Y}, \\
\tilde{X} & =e^{K / 2} \sqrt[4]{\frac{1+\alpha_{2}^{2}}{1+\alpha_{1}^{2}}} X, \tilde{Y}=e^{-K / 2} \sqrt[4]{\frac{1+\alpha_{1}^{2}}{1+\alpha_{2}^{2}}} Y .
\end{aligned}
$$

The two oscillators of (31) share the same mass $m=$ $1 / \sqrt{\left(1+\alpha_{1}^{2}\right)\left(1+\alpha_{2}^{2}\right)}$, and have the frequencies

$$
\begin{gathered}
\Omega e^{K}=\sqrt{1 / 2\left[\left(C_{1}^{2}+C_{2}^{2}\right)-\sqrt{\left(C_{1}^{2}-C_{2}^{2}\right)^{2}-C_{3}^{2}}\right]}, \\
\Omega e^{-K}=\sqrt{1 / 2\left[\left(C_{1}^{2}+C_{2}^{2}\right)+\sqrt{\left(C_{1}^{2}-C_{2}^{2}\right)^{2}-C_{3}^{2}}\right]} .
\end{gathered}
$$

The explicit form of the parameters $\Omega$ and $K$ can be found from (34) as $\Omega=\sqrt[4]{\left(C_{1}^{2} C_{2}^{2}+C_{3}^{2} / 4\right)}$ and $e^{K}=$ $\sqrt{\frac{\left[\left(C_{1}^{2}+C_{2}^{2}\right)-\sqrt{\left(C_{1}^{2}-C_{2}^{2}\right)^{2}-C_{3}^{2}}\right]}{\left[\left(C_{1}^{2}+C_{2}^{2}\right)+\sqrt{\left(C_{1}^{2}-C_{2}^{2}\right)^{2}-C_{3}^{2}}\right.}}$. Note that in oreder to keep two frequencies of (34) real, the condition $\left|C_{1}^{2}-C_{2}^{2}\right| \geq$ $C_{3}$ needs to be satisfied. Thus (31) is an anisotropic oscillator and its eigenvalues are [17]

$$
E=\Omega\left[e^{K}\left(n_{K}+1 / 2\right)+e^{-K}\left(n_{-K}+1 / 2\right)\right],
$$

where $n_{K}, n_{-K} \in \mathbb{N}$. Before discussing the $\mathcal{P} \mathcal{T}$ symmetric noncommutative quantum mechanics for (31), we first discuss the problem in standard noncommutative space. We consider only coordinates to be noncommutative of the form

$$
[\hat{\tilde{X}}, \hat{\tilde{Y}}]=2 i \underline{\theta}
$$

with the representation $\hat{\tilde{X}}=\tilde{X}-\underline{\theta} \tilde{P_{Y}}, \hat{\tilde{Y}}=\tilde{Y}+\underline{\theta} \tilde{P_{X}}$. Note that consistency with (2) and (41) demands that $\underline{\theta}=\left(\alpha_{1}-\alpha_{2}\right) \theta$. The noncommutative version of (31) is

$$
\begin{aligned}
H & =e^{K} H_{K}+e^{-K} H_{-K} \\
& +e^{K}\left(m^{2} \Omega^{2} \underline{\theta}^{2}{\tilde{P_{Y}}}^{2}-m \Omega^{2} \underline{\theta} \tilde{X} \tilde{P_{Y}}\right) \\
& +e^{-K}\left(m^{2} \Omega^{2} \underline{\theta}^{2}{\tilde{P_{X}}}^{2}+m \Omega^{2} \underline{\theta} \tilde{Y} \tilde{P_{X}}\right)
\end{aligned}
$$

The perturbative spectrum of (37) is given by

$$
\begin{aligned}
E & =\Omega\left[e^{K}\left(n_{K}+1 / 2\right)+e^{-K}\left(n_{-K}+1 / 2\right)\right] \\
& +\Omega^{3} m^{3} \underline{\theta}^{2}\left[e^{K}\left(n_{K}+1 / 2\right)+e^{-K}\left(n_{-K}+1 / 2\right)\right](38)
\end{aligned}
$$

Now let us consider the $\mathcal{P} \mathcal{T}$-symmetric noncommutative case. In noncommutative phase space, the coordinates $X, Y, P_{X}, P_{Y}$ of (31) are replaced by the corresponding noncommutative counterparts $\hat{X}, \hat{Y}, \hat{P_{X}}, \hat{P_{Y}}$, which lead to

$$
\begin{aligned}
& \hat{H}=e^{K} H_{K}+e^{-K} H_{-K} \\
&-e^{K}\left(\frac{\underline{\hat{\theta}}^{2}}{2 m} \tilde{Y}^{2}+\frac{m \Omega^{2} \underline{\theta}^{2}}{2}{\tilde{P_{Y}}}^{2}+i \underline{\hat{\theta}} m \tilde{Y} \tilde{P_{X}}-i m \Omega^{2} \underline{\theta} \tilde{X} \tilde{P_{Y}}\right) \\
&\left.-e^{-K}\left(\frac{\underline{\hat{\theta}}^{2}}{2 m} \tilde{X}^{2}+\frac{m \Omega^{2} \underline{\theta}^{2}}{2}{\tilde{P_{X}}}^{2}-i \underline{\hat{\theta}} m \tilde{X} \tilde{P_{Y}}+i m \Omega^{2} \underline{\theta} \tilde{Y_{X}} \tilde{P_{X}}\right) 39\right)
\end{aligned}
$$

Note that the noncommutative operators $\hat{X}, \hat{Y}, \hat{P_{X}}, \hat{P_{Y}}$, now satisfy the commutation algebra

$$
\begin{aligned}
{[\hat{X}, \hat{Y}] } & =-2 \underline{\theta},\left[\hat{P_{X}}, \hat{P_{Y}}\right]=-2 \hat{\theta}, \\
{\left[\hat{X}, \hat{P_{X}}\right] } & =\left[\hat{P}, \hat{P_{Y}}\right]=i \hat{\hbar},
\end{aligned}
$$

where $\underline{\theta}=\left(\alpha_{1}-\alpha_{2}\right) \theta, \underline{\hat{\theta}}=\hat{\theta} /\left(\alpha_{1}-\alpha_{2}\right)$ and the representation

$$
\begin{aligned}
\hat{X} & =X+i \underline{\theta} P_{Y}, \hat{Y}=Y-i \underline{\theta} P_{X} \\
\hat{P_{X}} & =P_{X}-i \underline{\hat{\theta}} Y, \hat{P_{Y}}=P_{Y}+i \underline{\hat{\theta}} X,
\end{aligned}
$$

which can be obtained from (7), is used to get (39). It is possible to solve (39) by perturbation. The eigenvalues are given by

$$
\begin{aligned}
E & =\Omega\left[e^{K}\left(n_{K}+1 / 2\right)+e^{-K}\left(n_{-K}+1 / 2\right)\right] \\
& +\frac{1}{2} \Omega^{3} m^{2} \underline{\theta}^{2}\left[e^{K}\left(n_{K}+1 / 2\right)+e^{-K}\left(n_{-K}+1 / 2\right)\right] \\
& +\frac{1}{2} \frac{\hat{\theta}^{2}}{m^{2} \Omega}\left[e^{K}\left(n_{K}+1 / 2\right)+e^{-K}\left(n_{-K}+1 / 2\right)\right] .(42)
\end{aligned}
$$

Note that all the complex terms of (39) has the zero expectation values, which makes the spectrum (42) real. Importantly, as mentioned before, the condition for reality of frequencies, $\left|C_{1}^{2}-C_{2}^{2}\right| \geq C_{3}$, must have to be satisfied by (38) and (42) in order to keep the spectrum real. Othewise, it will generate complex eigenvalues in conjugate pairs.

\section{CONCLUSION AND DISCUSSION}

In this article $\mathcal{P} \mathcal{T}$-symmetric quantum mechanical model is studied in noncommutative space. In order to keep the $\mathcal{P} \mathcal{T}$-symmetry intact, we needed a non-commutative formulation which itself respects $\mathcal{P} \mathcal{T}$-symmetry. This is done by replacing the noncommutativity parameter with pure imaginary parameter. However the systems on the $\mathcal{P} \mathcal{T}$-symmetric noncommutative spaces generates complex eigen-values in some cases. On the other hand non Hermitian systems in standard noncommutative space has been found to have entirely real spectrum. 
[1] M. R. Douglas and N. A. Nekrasov, Rev. Mod. Phys. 73, 977 (2001).

[2] A. P. Balachandran, T. R. Govindarajan, C. Molina and P. Teotonio-Sobrinho, JHEP 0410 (2004) 072.

[3] Jian-zu Zhang, Phys. Rev. Lett. 93, 043002 (2004).

[4] V.P. Nair and A.P. Polychronakos, Phys.Lett. B505, 267 (2001).

[5] P. K. Ghosh, Eur. Phys. J. C42,355 (2005).

[6] P. D. Alvarez, J. Gomis, K. Kamimura and M. S. Plyushchay, Phys. Lett. B659, 906 (2008).

[7] O. Bertolami, J. G. Rosa, C. M. L. de Aragao, P. Castorina and D. Zappala, Phys. Rev. D72, 025010 (2005).

[8] A. Kijanka and P. Kosinski, Phys. Rev. D70, 127702 (2004).
[9] P. R. Giri and P. Roy, arXiv:0803.4090v2 [hep-th].

[10] C. Bender and S. Boettcher, Phys. Rev. Lett. 80, 5243 (1998)

[11] C. Bender, Rept. Prog. Phys 70, 947 (2007).

[12] C. M Bender, D. C Brody and H. F Jones, Phys.rev.Lett 89, 270401 (2002).

[13] A. Mostafazadeh, Phys. Rev. Lett. 99, 130502 (2007).

[14] K.G. Makris, R. El-Ganainy , D.N. Christodoulides and Z.H. Musslimani, Phys. Rev. Lett. 100103904

[15] K.G. Makris, R. El-Ganainy , D.N. Christodoulides and Z.H. Musslimani, Phys. Rev. Lett. 100, 103904 (2008).

[16] M. M. Sheikh-Jabbari, Phys. Rev. Lett. 84, 5265 (2000).

[17] A. Nanayakkara, Phys. Lett. A304, 67 (2002). 